\title{
Homodimers of Chromosomal Proteins HMG-14 and HMG-17 in Nucleosome Cores
}

\section{Yuri V. Postnikov*, Lothar Trieschmann, Anke Rickers and Michael Bustin}

Laboratory of Molecular Carcinogenesis, National Cancer Institute, National Institutes of Health, Bethesda MD 20892, USA

*Corresponding author
In this work, we report that nucleosome core particles interact with an equimolar mixture of the chromosomal proteins HMG-14 and HMG-17 to form, exclusively, complexes containing two molecules of either HMG-14 or HMG-17 (homodimers). Analysis of the binding of various mixtures of wild-type proteins and their deletion mutants indicates that homodimer formation is not dependent on contacts between the nucleosome-bound HMG-14/-17 proteins themselves. We suggest that HMG-14/-17 proteins in nucleosomes cross-talk by inducing specific allosteric transitions in the chromatin subunit.

(C) 1995 Academic Press Limited

\section{Introduction}

The chromosomal proteins HMG-14 and HMG-17 are found in the nuclei of all higher eukaryotes, and may be involved in the generation or maintenance of structural features which are unique to transcriptionally active chromatin (reviewed in Bustin et al. (1990b)). The intron-exon organization of the genes coding for the two proteins is similar, suggesting that they evolved from a common ancestor (Bustin et al., $1990 \mathrm{~b}$ ). Indeed, over $40 \%$ of the amino acid positions in the HMG-14/-17 protein family are invariant and the two protein groups are similar in many respects, including molecular mass, net charge, conformation and interactions with DNA and chromatin (Bustin et al., 1990b; Johns, 1982). They footprint to the same position in nucleosomes (Alfonso et al., 1994), have similar effects on the structure of nucleosomes, and both can enhance the transcriptional potential of chromatin assembled in cell free extracts (Crippa et al., 1993; Trieschmann et al., 1995). These similarities suggest that HMG-14 and HMG-17 may also have similar, or perhaps even identical, cellular functions. However, all cells from higher eukaryotes invariably contain both HMG-14 and HMG-17 protein, suggesting that both proteins are necessary for proper cellular function and that the structural differences between the proteins, although not readily apparent, may be functionally significant.

Abbreviations used: $\mathrm{CP}$, core particles; HMG, HMG proteins; PCR, polymerase chain reaction; TBE, Tris, boric acid, EDTA.
Structural differences between HMG-14 and HMG17 can be detected by comparing their hydropathy index profile and studying their immunological specificity (Landsman \& Bustin, 1986). The two proteins also differ in the net negative charge density of their C-terminal regions and in the proline content of their nucleosomal binding domain (Bustin et al., 1990b; Landsman \& Bustin, 1986). Small differences in their binding sites on nucleosome cores have been detected (Shick et al., 1985). Functional differences between the proteins are also suggested by the recent observation that the mitogenic induction of immediate early genes is accompanied by the phosphorylation of HMG-14, but not HMG-17 (Barrat et al., 1994).

Although the cellular function of the HMG-14/-17 proteins is not fully understood, it is very likely that the function of these proteins depends on proper interaction with nucleosomes in chromatin. HMG-14 and HMG-17 are the only known nuclear proteins which specifically recognize the 146 base-pair nucleosomal core particle (Albright et al., 1980; Mardian et al., 1980; Sandeen et al., 1980). Both proteins bind to nucleosome cores without any specificity for the underlying DNA sequence, suggesting that they recognize structural features specific to the nucleosomal core particles. Depending on the ionic strength, the proteins bind either cooperatively or non-cooperatively to nucleosome cores and, at appropriate HMG:nucleosome molar ratios, yield complexes containing two molecules of HMG-14/-17 per core particle (Sandeen et al., 1980). Both proteins bind symmetrically and interact with the DNA in the two major grooves flanking the 
nucleosomal dyad axis and in the region which is 20 to 25 base-pairs from the end of the core particle (Alfonso et al., 1994; Mardian et al., 1980; Sandeen et al., 1980).

Recently, we suggested that in the nucleus, HMG-14/-17 proteins bind to nucleosomes in a cooperative manner (Postnikov et al., 1994). The question arises whether the cooperative binding is random, producing all possible combinations of the homo- and heterodimers of HMG-14 and HMG-17, or whether the interaction is restricted to produce only one type of complex. Here, we show that HMG-14 and HMG-17 bind to nucleosomes to form, exclusively, homodimers containing either two molecules of HMG-14 or two molecules of HMG-17. Studies with deletion mutants suggest that the nucleosomal binding domain of the proteins induces specific allosteric transitions in the nucleosome cores, leading to the formation of complexes containing only homodimers of these HMG proteins.

\section{Results}

Potentially, the interaction between a mixture of HMG-14 and HMG-17 proteins and nucleosome cores may result in the formation of complexes containing only homodimers of each protein, complexes containing only heterodimers, or a random mixture of all possible combinations. To distinguish between these three possibilities, it is necessary to separate nucleosomes complexed with HMG-14 from those complexed with HMG-17. Nucleosome-HMG-14 complexes have the same electrophoretic mobility as nucleosome-HMG-17 complexes; therefore, we have used specific antibodies to fractionate the two types of complexes, and two-dimensional gel analysis to identify the various proteins in the core-HMG complexes. In these experiments (Figure 1), equimolar amounts of HMG-14 and -17 proteins were incubated with nucleosome core particles, and the resulting complexes treated with antibodies specific to one of the HMG proteins. These reaction mixtures contained three major components: free nucleosome core particles $(\mathrm{CP})$, core particles complexed with HMG proteins (CP + HMG), and complexes of IgG molecules bound to the $\mathrm{CP}+\mathrm{HMG}$ complex $(\mathrm{CP}+\mathrm{HMG}+\mathrm{IgG})$. Electrophoresis on native polyacrylamide gels resolved the three components in the reaction mixture, while SDS-PAGE in the second dimension resolved the various proteins present in each complex.

\section{The cooperative binding of the HMG-14/-17 protein to nucleosome cores exclusively produces homodimer complexes}

The antibodies used in these studies were elicited against purified proteins. Analysis by ELISA, Western blots and radioimmunoassay indicated that each sera reacted specifically with the immunogen (Bustin, 1989). We tested whether the antibodies elicited against the free protein will also specifically

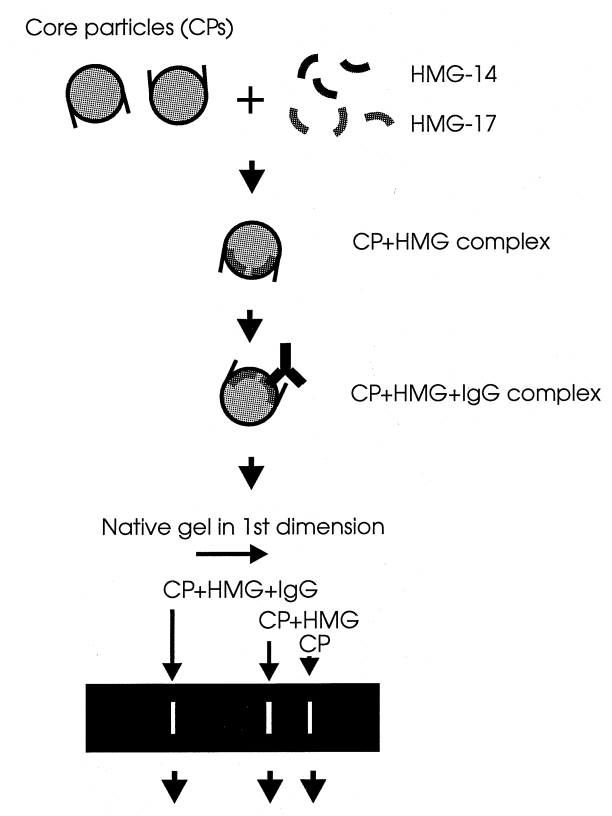

Protein SDS gel in 2nd dimension

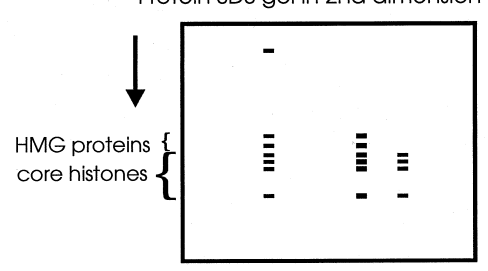

Figure 1. Experimental protocol used to determine whether HMG-14/-17 bind to nucleosomes as homo- or heterodimers. Core particles were incubated with equimolar mixtures of HMG-14 and HMG-17 to produce $\mathrm{CP}+\mathrm{HMG}$ complexes. Immunoaffinity purified antibodies were added to form the CP + HMG + IgG complex. The complexes were fractionated in the first dimension on native polyacrylamide gels and the proteins in the various complexes resolved by electrophoresis, in SDS-containing gels, in the second dimension.

recognize the nucleosome bound HMGs. Nucleosome cores were complexed with either HMG-14 or HMG-17 and each complex was reacted with antibodies against each of the proteins. Analysis of the reaction mixture by electrophoresis on native polyacrylamide gels (Figure 2) revealed that both anti-HMG-14 and anti-HMG-17 antibodies react specifically with the corresponding HMG protein bound to nucleosome cores, and that the CP + HMG + IgG complex can be easily separated from the $\mathrm{CP}+\mathrm{HMG}$ complex. In these experiments, we observed that the number of the supershifted bands was related to the ratio of antibodies to nucleosome in the reaction mixture, and that an excess of antibodies leads to formation of additional bands and large aggregates which interfere with the analysis of the results. We assumed that the additional bands correspond to complexes containing more than one antibody molecule per nucleosome. Therefore, the amount of antibodies added was chosen to minimize the formation of large 


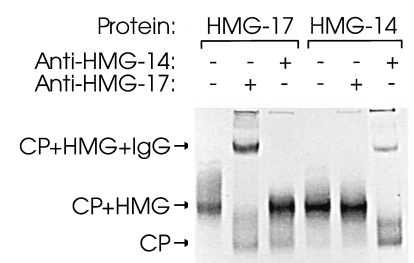

Figure 2. Specificity of the antibody supershift assay for the detection of nucleosome-bound HMG-14/-17 protein. Core particles $(\mathrm{CP})$ were reconstituted under cooperative binding conditions with either HMG-17 or HMG-14 at a molar ratio of $1: 2$ and treated with antibodies against either HMG-14 (antiHMG-14) or HMG-17 (antiHMG-17). The resulting complexes $(\mathrm{CP}, \mathrm{CP}+\mathrm{HMG}$ and $\mathrm{CP}+$ $\mathrm{HMG}+\mathrm{IgG})$ were resolved on native $5 \%(\mathrm{w} / \mathrm{v})$ polyacrylamide gels and stained with ethidium bromide. The mobility of the various complexes is indicated on the left.

aggregates, and frequently supershifted only part of the nucleosome complexes.

In these experiments, it was especially important to ensure that the antibodies bound to nucleosomes containing two molecules of HMG protein, and that antibody binding did not cause a preferential release of a heterologous HMG from the nucleosome. Therefore, we examined the mobility of mixtures containing free core particles, core particles with one molecule of HMG, and core particles with two molecules of HMG (formed under non-cooperative binding conditions), in native gels run under cooperative conditions. The results clearly indicate that under cooperative conditions, only two molecular species exist: one corresponding to the free core particle, and one corresponding to a core particle with two HMG molecules. Core particles complexed with only one molecule of HMG do not exist under co-operative binding conditions (data not shown).

To determine whether HMG-14/-17 bind to nucleosomes as homo- or heterodimers, nucleosome cores were added to an equimolar premix of HMG-14 and HMG-17 in $2 \times$ Tris, boric acid, EDTA (TBE) buffer, i.e. under cooperative binding conditions. After the addition of antibodies, the complexes were resolved in the first dimension, and the protein content of each complex resolved by SDS-PAGE in the second dimension (Figure 3). Examination of the two-dimensional gels indicated that the nucleosome cores which were supershifted by the affinity pure antibodies contained only one type of HMG. Thus, supershifts produced with anti-HMG-14 antibodies (the band indicated as CP + HMG + antiHMG14) contained only HMG-14 protein and were devoid of HMG-17 (Figure 3A), while the bands supershifted by antibodies to HMG-17 contained HMG-17 and were devoid of HMG-14 (Figure 3B). HMG-17 protein was not detected in the nucleosomes which did not shift (band $\mathrm{CP}+\mathrm{HMG}$, Figure 3B), suggesting that the antibodies removed all the HMG-17 containing nucleosomes. In the case of HMG-14, not all the protein was removed, suggesting that the titer of the

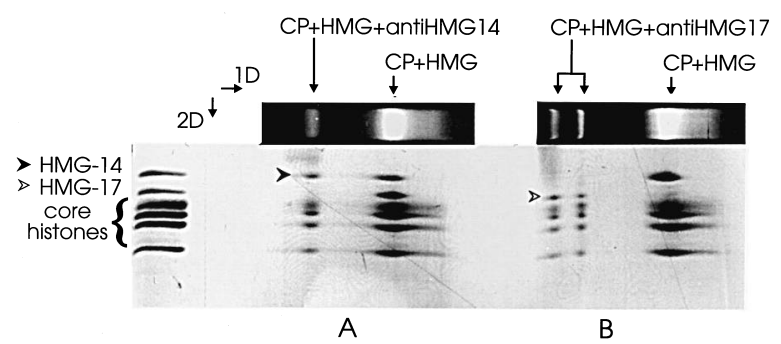

Figure 3. The cooperative binding of $\mathrm{HMG}-14 /-17$ to nucleosome cores leads to the formation of homodimers. Core particles $(\mathrm{CP})$ were reconstituted in $2 \times \mathrm{TBE}$ with a premix of HMG-17 and HMG-14 at a molar ratio of 1:1:1, incubated with antibodies against either HMG-14 (anti-HMG-14, gel A) or HMG-17 (antiHMG-17, gel B). The resulting complexes were analyzed by two-dimensional gel electrophoresis. These were separated on a $5 \%$ polyacrylamide gel containing $2 \times \mathrm{TBE}$ in the first dimension and stained with ethidium bromide (black strips on the top of the gel). The protein composition of the various complexes was visualized by SDS 10-20\% (w/v) PAGE, with subsequent silver staining. The migration of the markers is indicated on the left.

anti HMG-14 antibodies is lower than that of the anti HMG-17 antibodies.The anti HMG-17 antibodies produced two supershifts, most probably complexes containing either one or two antibody molecules. Although the silver staining method is not quantitative, a comparison with the protein stain in the marker lane indicated that, in the various complexes, the amount of HMG-14 or HMG-17 was approximately equivalent to that of the histones. To further examine this theory, the experiments were scaled up and the proteins detected by Coomassie blue. The results indicated that the amount of HMG-14 or HMG-17 in the antibody supershifted bands was indeed equivalent to that of the core histones (data not shown). To avoid the possibility that the interaction of an antibody with a nucleosome-bound HMG releases the second HMG molecule, the experiments were repeated with formaldehyde-fixed HMG-nucleosome complexes. Examination of these complexes, after reversal of the cross-links, also indicated that the HMG proteins segregate and bind to the nucleosomes as homodimers. These results suggest that all nucleosomes which were shifted did indeed contain two molecules of either the HMG-14 or HMG-17 protein.

We conclude, therefore, that, under cooperative binding conditions, where each nucleosome contains two molecules of HMG-14/-17, these proteins reside on different nucleosomes; therefore, the nucleosome cores contain homodimers of either HMG-14 or HMG-17.

\section{Cross-talk between HMG-14/-17 homodimers on nucleosomes occurs via their nucleosomal binding domain}

The presence of nucleosomes containing only homodimers of either HMG-14 or HMG-17 proteins 


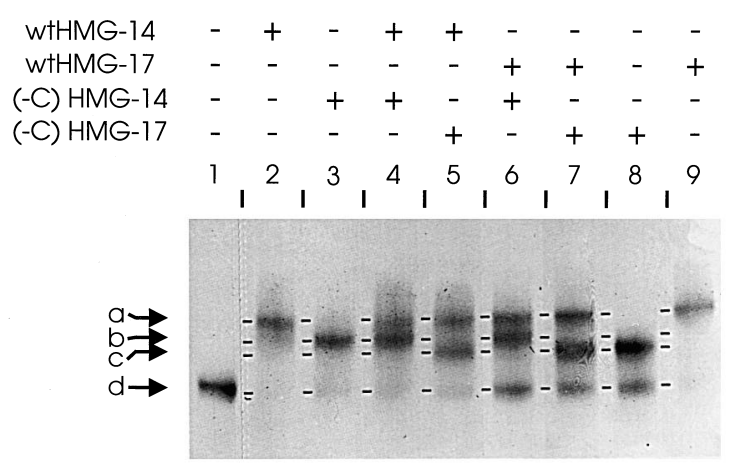

Figure 4. Homodimeric cooperative binding of wildtype HMG-14/-17 proteins and their C-terminal deletion mutants to nucleosome cores. Core particles were incubated under cooperative conditions with various combinations (as shown above the gel) of wild-type proteins (wtHMG-14 and -17) and their C-terminal truncation mutants ((-C)HMG-14 and -17), at a HMG:nucleosome molar ratio of 2 . Gel electrophoresis was done in $2 \times$ TBE. All reconstituted complexes had two HMG molecules per particle. CP + HMG-14 and CP + HMG-17 complexes have similar mobility and are lettered as band a, CP + (-C)HMG-14 as band b, CP + (-C)HMG-17 as band $\mathrm{c}$, and non-complexed $\mathrm{CP}$ as band $\mathrm{d}$.

was unexpected, since previous studies did not reveal any significant differences in the binding of these two proteins to nucleosome cores. The footprint of HMG-14/-17 on the nucleosomes suggested that the cooperative binding of the HMG-14/-17 protein to nucleosome cores may involve interactions between their C-terminal domains (Alfonso et al., 1994). Therefore, we produced C-terminal deletion mutants of both HMG-14 and HMG-17 and studied the binding of these mutants, as well as all the possible combinations of C-terminal deletion mutants and wild-type proteins, to nucleosome cores (Figure 4).

Under cooperative binding conditions, the electrophoretic mobility of the nucleosomes complexed with the C-terminal deletion mutant of HMG-14, (-C)HMG-14, is different from the complex containing $(-\mathrm{C}) \mathrm{HMG}-17$ and the complex containing either one of the wild-type proteins. An equimolar mixture of any kind of combination of wild-type and C-terminal mutant proteins invariably produced two bands; each band corresponded to the migration of the nucleosomes complexed with a dimer of one of the two proteins present in the mixture. Thus, in lane 4 an equimolar mixture of wild-type HMG-14 and its C-terminal deletion mutant produced bands $\mathrm{a}$ and $\mathrm{b}$, which contain nucleosomes complexed with either HMG-14 alone (lane 2) or with (-C)HMG-14 alone (lane 3). Likewise, lanes 5, 6 and 7, containing the other three possible combinations of proteins, also produced only two bands. Bands with intermediate mobilities, which would have been indicative of heterodimer formation, are not seen. Analysis of the protein content of the various complexes in lanes 4 to 7 by second dimension SDS gel electrophoresis confirmed that each of the bands

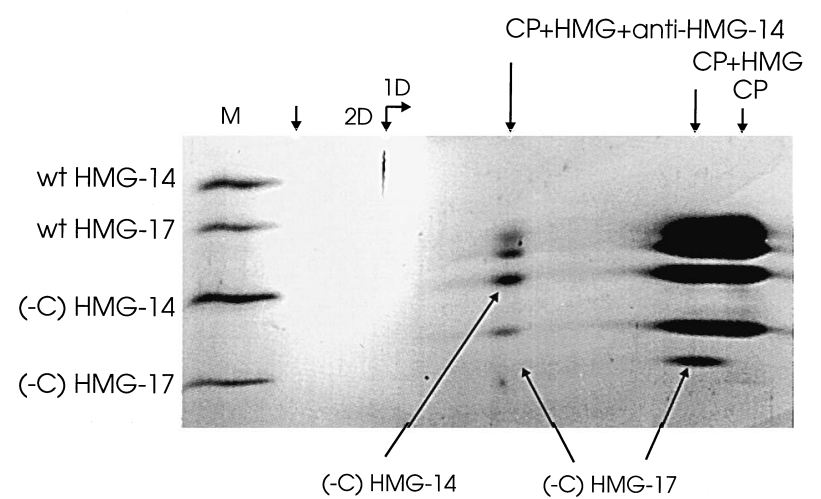

Figure 5. Homodimeric cooperative binding of Cterminal deletion mutants of HMG-14 and HMG-17 to nucleosome cores. Core particles $(\mathrm{CP})$ were incubated with a premix of C-terminal truncated variants of HMG-14 and HMG-17, (-C)HMG-14 and (-C)HMG-17, respectively, in $2 \times \mathrm{TBE}$ at a molar ratio of $1: 1: 1$, and incubated with immunoaffinity purified antibodies against a peptide corresponding to amino acids 42 to 57 of HMG-14. The complexes were analyzed by two-dimensional gel electrophoresis. Arrows above the gel indicate the mobility of the nucleoprotein complexes. The mobility of marker proteins, run only in the second dimension, is shown on the left. The mobility of $(-\mathrm{C}) \mathrm{HMG}-14$ is identical to that of histone $\mathrm{H} 2 \mathrm{~A}$, and therefore the supershifted particles seem to be enriched in this component. Note the absence of (-C)HMG-17 in the nucleosome cores supershifted by the HMG-14 specific antibodies.

contained only one type of HMG protein (data not shown).

One-dimensional electrophoresis does not resolve the core particles reconstituted with (-C)HMG-14 from those reconstituted with $(-\mathrm{C}) \mathrm{HMG}-17$ well (bands b and c in Figure 4). Therefore, we analyzed these complexes by two-dimensional electrophoresis, using an antibody elicited against a peptide corresponding to amino acid residues 42 to 57 of HMG-14 (Bustin et al., 1990). Previous studies demonstrated that this antibody recognizes the chromatin-bound HMG-14 protein (Bustin et al., 1990a). Mobility shift assays similar to those presented in Figure 1 indicated that these antibodies produce specific supershifts (not shown). Twodimensional gel analysis of a reaction mixture containing an equimolar mixture of core particles and the two C-terminal truncation mutants indicated that the band supershifted by HMG-14 specific antibodies (band CP + HMG + anti-HMG-14) was devoid of (-C)HMG-17. In contrast, the HMGnucleosome complexes (CP + HMG) which did not react with the antibodies contained significant amounts of the (-C)HMG-17 mutant (Figure 5). The mobility of the (-C)HMG-14 mutant is identical to that of histone $\mathrm{H} 2 \mathrm{~A}$, leading to an apparent increase in the relative intensity of the $\mathrm{H} 2 \mathrm{~A}$ histone band (as compared to other core histones). This result is consistent with the presence of significant amounts of $(-C) H M G-14$ in the supershifted band $(\mathrm{CP}+\mathrm{HMG}+\mathrm{IgG})$. In summary, under cooperative binding conditions, the $\mathrm{C}$-terminal deletion mutants 


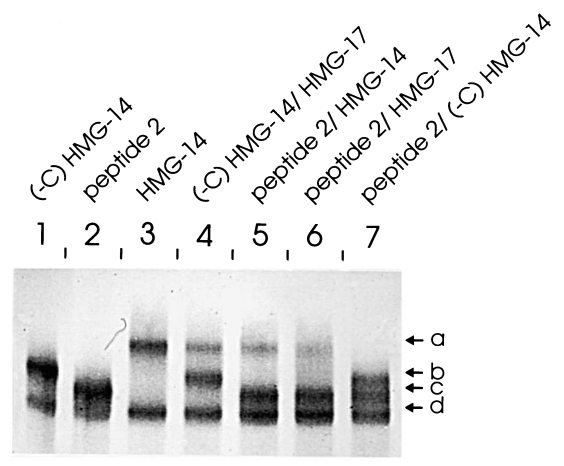

Figure 6. Minimal structural reguirements for homodimeric cooperative binding. Core particles were incubated under cooperative binding conditions with various HMG proteins as indicated above the lanes. Peptide 2 is a synthetic peptide, corresponding to the DNA-binding domain of HMG-17. The gel mobility shift assay was done as in Figure 4. The letters on the right side of the gel indicate the position of the various complexes. a, HMG-14 $+\mathrm{CP}$ or HMG-17 + CP; b, (-C)HMG-14 + CP; c, peptide 2 + CP; d, non-complexed CP. In lanes 5 and 6 the molar ratio of peptide to wild-type proteins was 2:1.

of both HMG-14 and HMG-17 proteins bind to nucleosomes to produce only homodimers. Likewise, the interaction of equimolar mixtures of any type of HMGs and their deletion mutants with nucleosome cores produces complexes containing only homodimers. We conclude, therefore, that the information necessary to produce homodimers does not reside in the C-terminal region of the proteins.

Most of the information necessary for the binding of the HMG-14/-17 proteins to nucleosomes resides in an evolutionarily conserved region defined as the nucleosomal binding domain of this protein family (Abercombie et al., 1978; Cook et al., 1989). A peptide (named peptide 2) corresponding to this region in HMG-17 (residues 17 to 46) retains some of the properties characteristic of the native protein and can act as an independent functional domain (Crippa et al., 1992). We therefore tested whether this region, by itself, also forms homodimers with nucleosomes. As indicated in Figure 6, nucleosomes complexed with peptide 2 (band c in lane 2) have an electrophoretic mobility which is different from that of nucleosomes complexed with (-C)HMG-14 (band b in lane 1) or nucleosomes complexed with intact HMG-14 (band a in lane 3). The various combinations of wild-type protein, their C-terminal deletion mutants, and peptide 2 all produced bands indicative of complexes containing nucleosomes with homodimers of the various components. Thus, peptide 2 did not produce complexes with intermediate mobility with either wild-type proteins (lanes 5 and 6) or with truncated HMG-14 (lane 7). In the presence of other proteins, peptide 2 bound to the core particle to produce a distinct band (band $\mathrm{c}$ in lanes 2, 5, 6, and 7). The finding that peptide 2 forms homodimers even in the presence of intact HMG-17 was unexpected. Therefore, we have performed additional experiments in which nu-

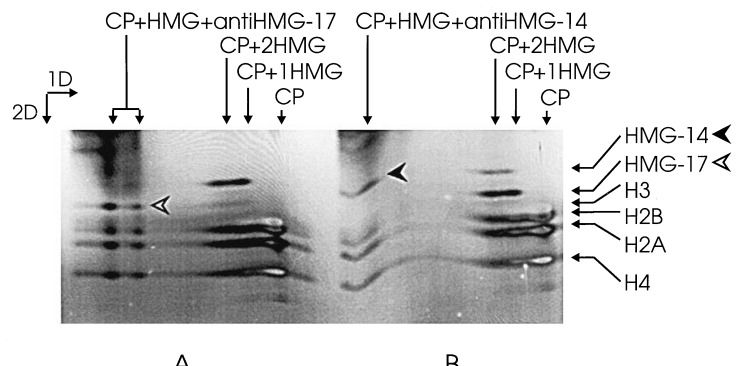

A

Figure 7. Non-cooperative binding of HMG-14/-17 to nucleosome cores leads to formation of homodimers. Core particles $(\mathrm{CP})$ were reconstituted in $0.2 \times \mathrm{TBE}$ with a premix of HMG-17 and HMG-14 at a molar ratio of 1:0.5:0.5, and incubated with antibodies against either HMG-17 (antiHMG-17, gel A) or HMG-14 (antiHMG-14, gel B) and analyzed by two-dimensional gel electrophoresis. The arrows at the top of the gel show the location of the nucleoprotein complexes after electrophoresis in the first direction. Proteins are marked HMG-14 (closed arrow), HMG-17 (open arrow) and core histones (see also Figure 3 for markers). The bands in the left upper area of each gel are the light chains of IgG.

cleosome cores were incubated with various solutions in which the molar ratio of peptide 2 to HMG-17 ranged from 0.5 to 2 . The results indicate that the formation of homodimers was independent of the molar ratio of the proteins, and in each case only homodimers of either HMG-17 or peptide 2 were formed (data not shown). Bands indicative of heterodimers were not observed. This situation is different from that occurring at low ionic strength, under non-cooperative binding conditions, where heterodimers are observed (Crippa et al., 1992).

Thus, the nucleosomal binding domain of HMG-17 by itself can form homodimers in nucleosome cores. This result is in agreement with previous findings, suggesting that this protein region can act as an independent functional domain (Crippa et al., 1992). It is important to note, however, that each deletion mutant, or wild-type protein, produced homodimers. Because heterodimers were not formed between homologous deletions and wildtype proteins, we suggest that other regions beside the nucleosomal binding domain participate in the interaction between HMG-14/-17 and the nucleosome cores.

\section{Full-length HMG proteins are required for the maintenance of the homodimeric mode of binding to core particles under non-cooperative conditions}

At low ionic strength (e.g. $0.2 \times$ TBE), proteins HMG-14/-17 bind to nucleosome core particles in a non-cooperative mode and, depending on the molar ratios of proteins to core particles, form complexes containing either one or two HMG molecules per nucleosome. Under non-cooperative binding conditions, as was the case under cooperative binding, the nucleosomes were associated with either 
A B

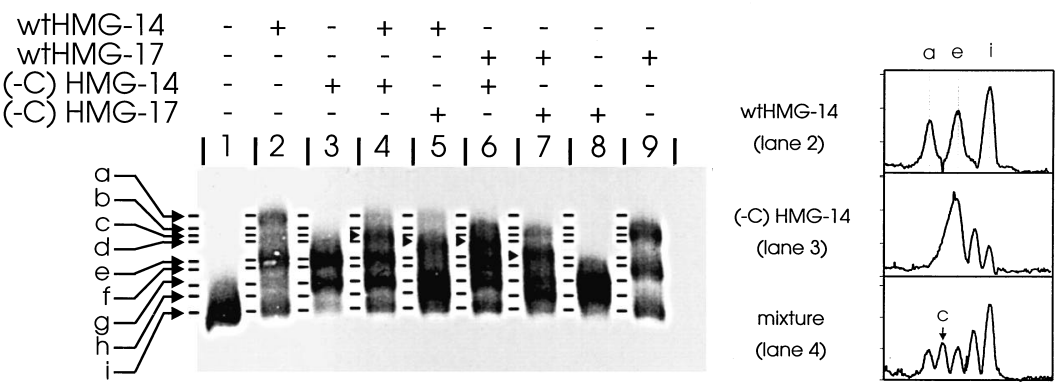

Figure 8. C-terminal deletion of HMG-14/-17 proteins leads to the formation of heterodimers under non-cooperative binding conditions. Core particles $(\mathrm{CP})$ were incubated under non-cooperative binding conditions $(0.2 \times$ TBE $)$ with wild-type proteins (wtHMG-14 and -17) and C-terminal truncation mutants $((-\mathrm{C}) \mathrm{HMG}-14$ and -17$)$ as indicated on top of the gel. A, Gel mobility shift assay of the complexes. Letters from a to $\mathrm{i}$ indicate the mobility of the following complexes (some of which cannot be resolved): a, CP + 2HMG-14; b, CP + 2HMG-17; c, CP + 1HMG-14 + 1(-C)HMG-14; d, CP + 1HMG$14+1(-\mathrm{C}) \mathrm{HMG}-17$ or CP + 1HMG-17 + 1(-C)HMG-14; e, CP + 1HMG-14 or CP + 2(-C)HMG-14; f, CP + 1HMG-17; g, $\mathrm{CP}+1(-\mathrm{C}) \mathrm{HMG}-14$ or CP $+2(-\mathrm{C}) \mathrm{HMG}-17$; h, CP + 1(-C)HMG-17; i, CP. Black triangles in lanes 4, 5, 6 and 7 (bands $\mathrm{C}$, $\mathrm{d}, \mathrm{d}$, and e, respectively) indicate heterodimers of HMG proteins bound to the core particle. B, Densitometric scans of lanes 2, 3 and 4 . The background was subtracted using tangent lines. The positions of peaks were strongly preserved in the various lanes. Peaks a, e, and i correspond to bands a, e and i in A. Peak c on the lower chart corresponds to band c, which contains the CP + 1HMG-14 + 1(-C)HMG-14 complex and is absent from lanes 2 and 3 (upper and middle scan).

HMG-14 or HMG-17, and complexes containing heterodimers were not detected (Figure 7). Thus, the band which was supershifted by antiHMG-17 IgG (band $\mathrm{CP}+2 \mathrm{HMG}+$ antiHMG-17 in Figure 7A) contained only HMG-17 protein, while the nonsupershifted bands $(\mathrm{CP}+2 \mathrm{HMG}$ and $\mathrm{CP}+1 \mathrm{HMG})$ were rich in HMG-14 protein. The converse was true when anti-HMG-14 antibodies were used to produce the supershift (Figure 7B). The supershifted band contained HMG-14 and was devoid of HMG-17, while the bands which did not supershift with the antibodies (i.e. $\mathrm{CP}+2 \mathrm{HMG}$ and $\mathrm{CP}+1 \mathrm{HMG}$ ) were rich in HMG-17.

However, under these non-cooperative binding conditions, deletion of the C-terminal region of either HMG-14 or HMG-17 led to the formation of heterodimers (Figure 8). For example, nucleosomes complexed with a mixture of HMG-14 and (-C)HMG-14 (Figure 8A, lane 4) formed band c (arrow in lane 4), which is not present in nucleosomes complexed with either HMG-14 alone (Figure 8A, lane 2) or (-C)HMG-14 alone (Figure 8, lane 3 ). The presence of this new band was verified by densitometric analysis of lanes 2 to 4 in Figure 8A (Figure 8B). Thus, this band corresponds to a heterodimer in which one binding site on the nucleosome core is occupied by HMG-14 and the other site is occupied by the HMG-14 C-terminal truncated mutant. Likewise, nucleosomes complexed was a mixture of HMG-14 and (-C)HMG-17 produced band d (Figure 8A, lane 5), which was not present in nucleosomes complexed with only one of these two proteins (see Figure 8A, lanes 2 and 8).

The results presented in Figure 8 strongly suggest that under non-cooperative binding conditions, truncated proteins form heterodimers in nucleosome cores. However, the one-dimensional electrophoretic pattern is very complex and some of the bands overlap and cannot be easily resolved. Two-dimensional gel analysis (Figure 9) resolved all the molecular species except the C-terminal truncation of HMG-14, which co-migrates with histone H2A, and therefore, its presence could not be unequivocally demonstrated. Therefore, the unequivocal presence of heterodimers is apparent only in panels $\mathrm{B}$ and $\mathrm{D}$ of Figure 9. In panel B, band d contains histones and both HMG-14 and the C-terminal truncation of HMG-17. This band has the same mobility as that of nucleosomes complexed with a heterodimer of HMG-17 and the C-terminal truncation of HMG-14 (band d panel C). Band e in panel D demonstrates the presence of a nucleosome complexed with a heterodimer of HMG-17 and the C-terminal truncation of HMG-17. Analysis of the various combinations of proteins indicates that, under non-cooperative binding conditions, all the C-terminal truncation mutants formed all the possible combinations of heterodimers (see arrows in Figure 8A). Detection of heterodimers by the methods used here adds further credence to the conclusion that, under cooperative binding conditions, only nucleosomes containing homodimers are formed.

We conclude, therefore, that under non-cooperative binding conditions, the presence of the C-terminal region of HMG-14 and HMG-17 is necessary for the formation of homodimers. Deletion of this region results in random binding, leading to nucleosome complexes containing protein heterodimers.

\section{Discussion}

The major finding reported in this manuscript is that the chromosomal proteins HMG-14 and HMG-17 bind to nucleosome cores to form, exclusively, complexes containing homodimers of either HMG-14 or HMG-17. At ionic strengths which are close to physiological conditions, C-terminal deletion mutants and even the isolated nucleosomal binding domain of HMG-17 bind as homodimers, suggesting that the underlying mechanism for homodimer formation does not depend on an interaction between the $\mathrm{HMG}-14 /-17$ proteins 


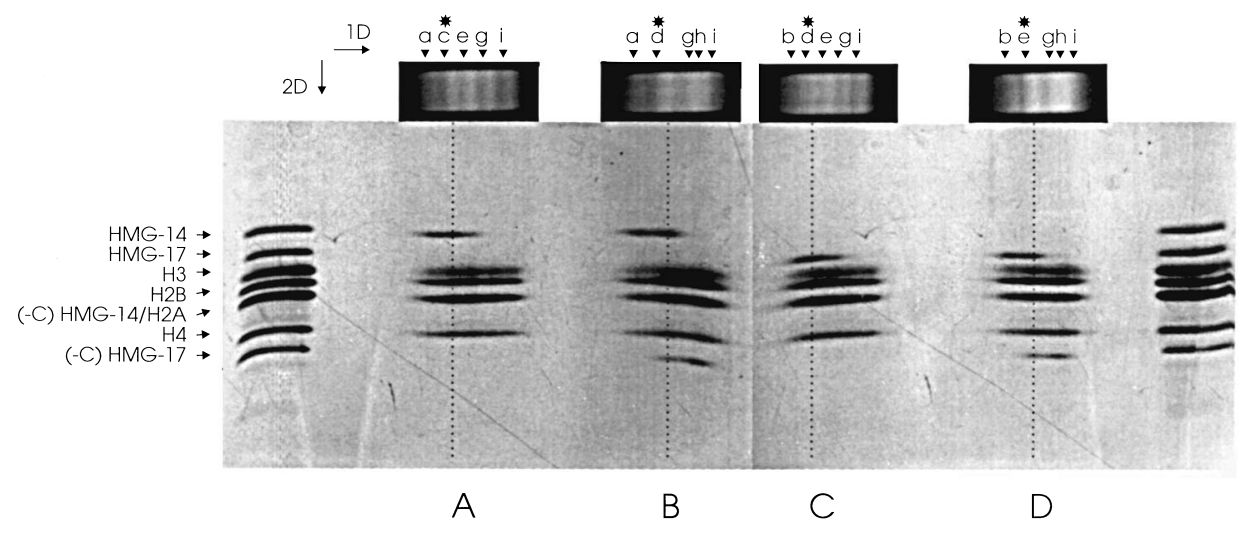

Figure 9. 2D analysis of heterodimers formed under non-cooperative binding conditions by wild-type HMG14/-17proteins and their C-terminal deletion mutants with nucleosome cores. Two-dimensional analysis of the core particles incubated under non-cooperative binding conditions $(0.2 \times \mathrm{TBE})$ with different mixtures of wild-type proteins (wtHMG-14 and -17) and C-terminal truncation mutants ((-C)HMG-14 and -17). Protein composition of tentative heterodimeric HMG-nucleosome complexes (lanes 4 to 7 in Figure 8A) was revealed by SDS gel electrophoresis in the second dimension. The mobility of diferent HMG-nucleosome complexes is depicted in lower case letters, as in Figure 8A. Tentative heterodimeric complexes are marked with asterisks. A, Lane $4(\mathrm{HMG}-14+(-\mathrm{C}) \mathrm{HMG}-14+\mathrm{CP})$. B, Lane 5 (HMG-14 + (-C)HMG-17 + CP). C, Lane 6 (HMG-17 + (-C)HMG-14 + CP). D, Lane 7 (HMG-17 + (-C)HMG-17 + CP).

themselves. We therefore suggest that these proteins induce specific allosteric transitions in nucleosomes, resulting in a strong preference for the formation of complexes containing HMG homodimers.

\section{Binding of HMG to nucleosomes}

It is generally accepted that chromatin structure is a key component of transcriptional regulatory mechanisms, and that changes in the transcriptional activity of genes often are accompanied by detectable changes in their chromatin structure. Studies on the molecular mechanisms involved in the generation of transcriptionally active chromatin suggest that the transcription potential of the chromatin template may, in part, be determined during nucleosome assembly on replicating DNA (Almouzni et al., 1990; Svaren \& Chalkley, 1990). Most of the studies on this subject have focused on histones; however, recent studies suggest that the kinetics of assembly of HMG-14/-17 non-histone proteins into chromatin also influence the transcription potential of the resulting template. In Xenopus egg extracts, the incorporation of HMG-14/-17 into nucleosomes during, but not after, chromatin assembly enhances the transcriptional potential of the resulting template (Crippa et al., 1993; Trieschmann et al., 1995). Thus, studies on the interaction of HMG-14/-17 proteins with chromatin may provide insights into processes linked to the generation of active chromatin.

At ionic strengths which are close to physiological, the interaction of HMG-14 or HMG-17 with chromatin subunits is cooperative, resulting in complexes containing two molecules of HMG-14/-17 per core particle (Sandeen et al., 1980). These results, and the recent analysis of the interaction of HMG-14 mutants with nucleosome cores, suggest that the cooperative interaction whereby two molecules of protein bind to the nucleosome is the predominant mode of HMG-14/-17 binding in cellular chromatin (Postnikov et al., 1994). Both HMG-14 and HMG-17 proteins are present in all tissues; therefore, cooperative interactions could lead to the formation of nucleosome complexes containing either homodimers, heterodimers, or a random mixture of these two proteins. So far, only the binding of a single HMG species to the nucleosome has been studied, and therefore the mode of binding of a mixture of proteins to nucleosome cores is not known. Our studies suggest that the proteins bind to nucleosomes to form homodimers exclusively.

What is the mechanism underlying the formation of nucleosomes containing homodimers of either HMG-14 or HMG-17? One possibility is that HMGs self-assemble to form homodimers and bind as such to the nucleosome core particles. This possibility is unlikely, since HMG-14/-17 dimers have never been detected and the purified proteins in aqueous solutions have very little ordered structure. Furthermore, it is difficult to conceive a mechanism whereby a preassembled HMG dimer would bind to nucleosomes in a symmetric fashion, producing multiple HMG-nucleosome contacts. Likewise, it is unlikely that the C-terminal truncations of the HMG proteins, and even the isolated nucleosomal binding domain, would also preassemble into homodimers and bind as such to core particles. A second explanation is that kinetic differences in the binding of the various proteins to the cores lead to the formation of homodimers on the nucleosomes. Under cooperative binding conditions, the dissociation constant for the binding of HMG-14 to nucleosome cores is approximately $1 \times 10^{-7}$ (Postnikov et al., 1994; Schroter \& Bode, 1982). We have recently determined 
that the dissociation constants for the binding of the HMG-14 C-terminal truncation mutant for wildtype HMG-17 and for the HMG-17 C-terminal truncation mutant are $1.2 \times 10^{-7}, 0.5 \times 10^{-7}$, and $0.5 \times 10^{-7}$, respectively (unpublished results). From kinetic considerations, it appears that these differences are too small to account for the exclusive formation of homodimers of these proteins on nucleosomes. A third possibility is that specific interactions between various domains of the nucleosome-bound HMG proteins stabilize the formation of homodimers. Indeed, the model for the location of HMG-14/-17 in nucleosomes is compatible with the possibility that the C-terminal regions of the nucleosome-bound HMGs are in close proximity (Alfonso et al., 1994). However, the finding that the C-terminal truncation mutants of both HMG-14 and HMG-17 and even a peptide corresponding to the nucleosomal binding domain of HMG-17 also form homodimers is not fully compatible with this possibility. Furthermore, HMG-histone rather than HMG-HMG contacts were detected in cross-linking experiments with chromatin (Brawley \& Martinson, 1992; Cook et al., 1986; Leffak \& Trempe, 1985).

We favor a fourth possibility, which assumes that the binding of the first HMG protein induces specific allosteric transition in the structure of the nucleosomes to create a binding site with greater affinity for the binding of a second, identical, molecule. The allosteric transitions are highly specific, and the structural changes induced by the nucleosomal binding domain are distinct from those induced by either the C-terminal truncation mutants or by the wild-type proteins. This suggestion is compatible with previous findings that the nucleosome binding region can act as an independent functional domain, and provides further support for the notion that HMG-14/-17 are modular proteins (Crippa et al., 1992). HMG-induced conformational changes in nucleosome cores have previously been considered as a possible explanation for an observed increase in the radius of gyration of nucleosome cores upon HMG binding (Uberbacher et al., 1982). The conformational transitions in nucleosomes involve only minor structural changes, since the proteins HMG-14/-17 also bind cooperatively to formaldehyde crosslinked nucleosome cores (Paton et al., 1983). These earlier findings are compatible with our suggestion that the binding of HMG to nucleosome cores involves precise and specific allosteric transitions. Allosteric transitions often involve short-range structural perturbations (Perutz, 1990) which are not always prevented by cross-linking (Kluger et al., 1994). In a complex multicomponent structure such as the nucleosome core particles, minor allosteric transitions may be difficult to detect.

The histone octamer is built of four symmetrical dimers which can easily undergo conformational transitions and may function as an "allosteric effector" (Arents \& Moudrianakis, 1993). It is therefore reasonable to assume that the multiple contacts between HMG proteins and both the DNA and the histones may induce allosteric transitions in the structure of the nucleosome particle. The specific allosteric transitions may involve only minor structural rearrangements similar in magnitude to those observed in other studies on HMG-nucleosome interactions (for numerous original references see Alfonso et al. (1994); Bustin et al. (1990)). We note that the homodimeric binding of HMG to nucleosomes at symmetrical sites would also preserve the overall symmetry of the protein components in the nucleosome particle.

In summary, these results suggest that the HMG proteins "cross talk" by inducing specific allosteric transitions in the nucleosome leading to the formation of homodimers. Thus, although HMG-14 and HMG-17 proteins are similar in many aspects, and produce identical footprints on the nucleosomal DNA, the structure of the nucleosome HMG-14 complex may be distinct from that of the nucleosome HMG-17 complex. The formation of specific HMG-nucleosome complexes may provide an explanation for the widespread occurrence of both HMG-14 and HMG-17 in most cells, and raises the possibility that, in the nucleus, each protein may be associated with nucleosomes containing a distinct subset of DNA sequences.

\section{Materials and Methods}

\section{Isolation of various HMG proteins and core particles}

Recombinant wild-type human HMG-14 and HMG-17 proteins and their C-terminal deletion mutants were expressed using the bacteriophage T7 RNA polymerase expression system and vector pVEX11 (a gift from A. Shankar, NIH) in BL21 (DE3) bacteria. Vectors for the expression of the deletion mutants were prepared by polymerase chain reaction (PCR) from the cDNAs coding for the wild-type proteins (Landsman et al., 1986a,b). The HMG-14 C-terminal deletion mutant lacked amino acids 78 to 99 , and the HMG-17 C-terminal deletion mutant lacked residues 59 to 89 . Purification of the recombinant proteins was carried out as described before (Bustin et al., 1991; Postnikov et al., 1994). Protein concentrations were determined by the BCA assay (Pierce). The molar concentration was calculated from the known amino acid composition, using the following molecular masses: wtHMG-14, 10,528 Da; wtHMG-17, $9261 \mathrm{Da}$; (-C)HMG-14, 8250 Da; (-C)HMG-17, 6250 Da; peptide 2, $3176 \mathrm{Da}$.

Nucleosome core particles were isolated from chicken red blood cells (Alfonso et al., 1994; Ausio et al., 1989). The size of the DNA in the nucleosome core particles used in this study was $146( \pm 2) \mathrm{bp}$. The histone content of the nucleosomes was monitored by electrophoresis in $15 \%(\mathrm{w} / \mathrm{v})$ polyacrylamide gels run in the presence of $0.1 \%(\mathrm{w} / \mathrm{v})$ SDS.

\section{Antibodies to HMG-14/-17 proteins}

Polyclonal rabbit antisera against HMG-14 and HMG-17 (Bustin et al., 1978) and polyclonal goat antiserum against peptide 5 (amino acids 42 to 57 of human HMG-14; Bustin et al., 1990a) were used as a source for various antibody 
preparations. Affinity pure antibodies were obtained with immunoaffinity columns, which were prepared by coupling pure recombinant human HMG-14 or HMG-17 protein to 3M Emphaze ${ }^{\text {tmk }}$ Biosupport Medium AB1 (Pierce) according to suppliers' recommendations. Immunoaffinity chromatography was carried out as follows: $10 \mathrm{ml}$ of antiserum was passed three times through $1.2 \mathrm{ml}$ columns containing immobilized antigen (5 to $8 \mathrm{mg} / \mathrm{ml}$ swollen gel) at room temperature at a flow rate of $0.2 \mathrm{ml} / \mathrm{min}$. The column was washed with 10 volumes of $1 \times$ PBS, $1 \%(\mathrm{v} / \mathrm{v})$ Tween-20 and 20 volumes of $1 \times$ PBS. The antibodies were eluted with $0.5 \mathrm{M} \mathrm{NaCl}(\mathrm{pH} 2.5)$, $12.5 \mathrm{ml} 0.1 \mathrm{M}$ glycine and immediately neutralized with $1 \mathrm{ml} 2 \mathrm{M}$ Tris ( $\mathrm{pH}$ 9.5). The antibody preparations were desalted and concentrated with Macrosep (Filtron Tech Corp) centrifugal concentrators (30 kDa MW cut off) to approximately $1 \mathrm{mg} / \mathrm{ml}$, and then sampled and stored at $-20^{\circ} \mathrm{C}$.

\section{Gel mobility shift assay}

Routinely, 5 pmol of core particles were incubated with 5 to 15 pmol of either a single protein species or an equimolar mixture of two HMG proteins in a volume of $10 \mu \mathrm{l}$, at $4^{\circ} \mathrm{C}$ for 10 minutes. For cooperative binding assays, the buffer was $2 \times \mathrm{TBE}$; for non-cooperative binding assays the buffer was $0.2 \times$ TBE $(1 \times$ TBE buffer $=100 \mathrm{mM}$ Tris (pH 8.4), $100 \mathrm{mM}$ borate, $1 \mathrm{mM}$ EDTA). For antibody mediated supershifts, $1 \mu \mathrm{l}$ affinity pure antibodies were added and the mixture incubated for 15 minutes at $4^{\circ} \mathrm{C}$. A $2 \mu \mathrm{l}$ volume of $20 \%$ (w/v) Ficoll- 400 was dispensed to each tube and the reaction mixture loaded on a $5 \%(\mathrm{w} / \mathrm{v})$ native polyacrylamide gel $(\% \mathrm{~T}=5, \% \mathrm{C}=5)$ containing either $2 \times$ or $0.2 \times \mathrm{TBE}$. The gels were prerun for one hour at $4^{\circ} \mathrm{C}$, run at $10 \mathrm{~mA}$ at $4^{\circ} \mathrm{C}$, and stained with ethidium bromide.

\section{Nucleosome/SDS polyacrylamide gradient two- dimensional gel}

After electrophoresis in the first dimension, the lanes of interest were excised and placed on top of the second dimension gel to determine the protein composition of the nucleosome-HMG complexes. The second dimension running gels were a 10 to $20 \%$ linear gradient of acrylamide (\% $\mathrm{C}=2.6$, for both light and heavy solutions). A $1 \mathrm{~cm}$ wide spacer gel $(\% \mathrm{~T}=5, \% \mathrm{C}=2.6)$ was layered and polymerized on top of the running gel. The gel strips from the first dimension were embedded and polymerized into a second layer of the stacking gel which was identical to the first stacking gel layer. Throughout the gel the buffer was 0.4 M Tris- $\mathrm{HCl}$ ( $\mathrm{pH}$ 8.8). A layer of SDS loading buffer was poured on top of the gel. The running buffer was $25 \mathrm{mM}$ Tris ( $\mathrm{pH} 8.3$ ), $190 \mathrm{mM}$ glycine, 0.1\% (w/v) SDS. For staining, the protein gel (1 mm thick) was rinsed with deionized water for 15 minutes, fixed with freshly prepared $10 \%$ glutaraldehyde (grade II, Sigma Chemical Co.) for 30 minutes, and washed at least five times with a large excess of water (20 minutes for each wash). Following this step, the procedure of Wray et al. (1981) was followed strictly. The gel was submerged in the ammoniacal silver solution for 20 minutes, washed with water for 10 minutes, and developed with freshly prepared formaldehyde/ citrate solution.

\section{Acknowledgement}

We thank Dr Roger Morton for a critical review of this manuscript.

\section{References}

Abercombie, B. D., Kneale, G. G., Crane-Robinson, C., Bradbury, E. M., Goodwin, G. H., Walker, J. M. \& Johns, E. W. (1978). Studies on the conformational properties of HMG-17 and its interaction with DNA. Eur. J. Biochem. 84, 173-177.

Albright, S. C., Wiseman, J. M., Lange, R. A. \& Garrard, W. T. (1980). Subunit structures of different electrophoretic forms of nucleosomes. J. Biol. Chem. 255, 3673-3684.

Alfonso, P. J., Crippa, M. P., Hayes, J. J. \& Bustin, M. (1994). The footprint of chromosomal proteins HMG-14 and HMG-17 on chromatin subunits. J. Mol. Biol. 236, 189-198.

Almouzni, G., Mechali, M. \& Wolfe, A. P. (1990). Competition between transcription complex assembly and chromatin assembly on replicating DNA. EMBO J. 9, 573-582.

Arents, G. \& Moudrianakis, E. N. (1993). Topography of the histone octamer surface: repeating structural motifs utilized in the docking of nucleosomal DNA. Proc. Natl Acad. Sci. USA, 90, 10489-10493.

Ausio, J., Dong, F. \& van Holde, K. E. (1989). Use of selectively trypsinized nucleosome core particles to analyze the role of histone "tails" in the stabilization of nucleosomes. J. Mol. Biol. 206, 451-463.

Barrat, J. M., Hazzalin, C. A., Cano, E. \& Mahadevan, L. C. (1994). Mitogen-stimulated phosphorylation of histone $\mathrm{H} 3$ is targeted to a small hyperacetylation-sensitive fraction. Proc. Natl Acad. Sci. USA, 91, 4781-4785.

Brawley, J. V. \& Martinson, H. G. (1992). HMG proteins 14 and 17 become cross-linked to the globular domain of histone $\mathrm{H} 3$ near the nucleosome core particle dyad. Biochemisty, 31, 364-370.

Bustin, M. (1989). Preparation and application of immunological probes for nucleosomes. Methods Enzymol. 170, 214-251.

Bustin, M., Hopkins, R. B. \& Isenberg, I. (1978). Immunological relatedness of high mobility group proteins from calf thymus. J. Biol. Chem. 253, 1694-1699.

Bustin, M., Crippa, M. P. \& Pash, J. M. (1990a). Immunochemical analysis of the exposure of high mobility group protein 14 and 17 surfaces in chromatin. J. Biol. Chem. 265, 20077-20080.

Bustin, M., Lehn, D. A. \& Landsman, D. (1990b). Structural features of the HMG chromosomal proteins and their genes. Biochim. Biophys. Acta, 1049, 231-243.

Bustin, M., Becerra, P. S., Crippa, M. P., Lehn, D. A., Pash, J. M. \& Shiloach, J. (1991). Recombinant human chromosomal proteins HMG-14 and HMG-17. Nucl. Acids Res. 19, 3115-3121.

Cook, G. R., Yau, P., Yasuda, H., Traut, R. R. \& Bradbury, E. M. (1986). High mobility group protein 17 cross-links primarily to histone $\mathrm{H} 2 \mathrm{~A}$ in the reconstituted HMG-17-nucleosome core particle complex. J. Biol. Chem. 261, 16185-16190.

Cook, G. R., Minch, M., Schroth, G. P. \& Bradbury, E. M. (1989). Analysis of the binding of high mobility group protein 17 to the nucleosome core particle by $1 \mathrm{HNMR}$ spectroscopy. J. Biol. Chem. 264, 1799-1803.

Crippa, M. P., Alfonso, P. J. \& Bustin, M. (1992). Nucleosome core binding region of chromosomal protein HMG-17 acts as an independent functional domain. J. Mol. Biol. 228, 442-449.

Crippa, M. P., Trieschmann, L., Alfonso, P. J., Wolfe, A. P. \& Bustin, M. (1993). Deposition of chromosomal protein HMG-17 during replication affects the 
nucleosomal ladder and transcriptional potential of nascent chromatin. EMBO J. 12, 3855-3864.

Johns, E. W. (1982). The HMG Chromosomal Proteins, Academic Press, London, UK.

Kluger, R., Jones, R. T. \& Shih, D. T. (1994). Cross linking hemoglobin by design. Artif. Cells Blood Substit. Immobil. Biotechnol. 22, 415-428.

Landsman, D. \& Bustin, M. (1986). Chromosomal proteins HMG-14 and HMG-17. Distinct multigene families coding for similar types of transcripts (published erratum appears in J. Biol. Chem. 1987, 262 (17): 8438). J. Biol. Chem. 261, 16087-16091.

Landsman, D., Soares, N., Gonzalez, F. J. \& Bustin, M. (1986a). Chromosomal protein HMG-17. Complete human cDNA sequence and evidence for a multigene family (published erratum appears in J. Biol. Chem. 1988, 263 (31): 16512). J. Biol. Chem. 261, 7479-7484.

Landsman, D., Srikantha, T., Westermann, R. \& Bustin, M. (1986b). Chromosomal protein HMG-14. Complete human cDNA sequence and evidence for a multigene family (published erratum appears in J. Biol. Chem. 1988, 263 (31): 16512). J. Biol. Chem. 261, 16082-16086.

Leffak, M. \& Trempe, J. P. (1985). Histone H1 and HMG $14 / 17$ are deposited nonrandomly in the nucleus. Nucl. Acids Res. 13, 4853-4869.

Mardian, J. K., Paton, A. E., Bunick, G. J. \& Olins, D. E. (1980). Nucleosome cores have two specific binding sites for nonhistone chromosomal proteins HMG 14 and HMG 17. Science, 209, 1534-1536.

Paton, A. E., Wilkinson-Singley, E. \& Olins, D. E. (1983). Nonhistone nuclear high mobility group proteins 14 and 17 stabilize nucleosome core particles. J. Biol. Chem. 258, 13221-13229.
Perutz, M. F. (1990). Mechanisms of Cooperativity and Allosteric Regulation in Proteins, Cambridge University Press, Cambridge, UK.

Postnikov, Y. V., Lehn, D. A., Robinson, R. C., Friedman, F. K., Shiloach, J. \& Bustin, M. (1994). The cooperative binding of chromosomal protein HMG-14 to nucleosome cores is reduced by single point mutations in the nucleosomal binding domain. Nucl. Acids Res. 22, 4520-4526.

Sandeen, G., Wood, W. \& Felsenfeld, G. (1980). The interaction of high mobility proteins $\mathrm{HMG}-14$ and -17 with nucleosomes. Nucl. Acids Res. 8, 3757-3778.

Schroter, H. \& Bode, J. (1982). The binding sites for large and small HMG proteins. Eur. J. Biochem. 127, $429-436$.

Shick, V. V., Belyavsky, A. V. \& Mirzabekov, A. D. (1985). Primary organization of nucleosomes. J. Mol. Biol. 185, 329-339.

Svaren, J. \& Chalkley, R. (1990). The structure and assembly of active chromatin. Trends Genet. 6, 52-56.

Trieschmann, L., Alfonso, P. J., Crippa, M. P., Wolfe, A. P. \& Bustin, M. (1995). Incorporation of chromosomal proteins HMG-14/-17 into nascent nucleosomes induces an extended chromatin conformation and enhances the utilization of active transcription complexes. EMBO J. 14, 1478-1489.

Uberbacher, E. C., Mardian, J. K. W., Rossi, R. M., Olins, D. \& Bunick, G. J. (1982). Neutron scattering studies and modeling the HMG-14 core nucleosome complex. Proc. Natl Acad. Sci. USA, 79, 5285-5262.

Wray, W., Boulikas, T., Wray, V. P. \& Hanckock, R. (1981). Silver staining of proteins in polyacrylamide gels. Anal. Biochem. 118, 197-203.

Edited by M. Yaniv 